\title{
Reply to: Nix and Lozada (2020), "Do police killings of unarmed persons really have spillover effects? Reanalyzing Bor et al. (2018)"
}

\author{
Jacob Bor ${ }^{1}$, Atheendar S. Venkataramani2 ${ }^{2 *}$, David Williams ${ }^{3}$, Alexander C. Tsai ${ }^{4}$
}

January 23,2020

${ }^{1}$ Boston University School of Public Health

${ }^{2}$ Perelman School of Medicine, University of Pennsylvania.*E-mail: atheenv@pennmedicine.upenn.edu

${ }^{3}$ Harvard T.H. Chan School of Public Health

${ }^{4}$ Massachusetts General Hospital and Harvard Medical School

We thank Courtney Boen, Mathew Kiang, Sandro Galea, Rourke O’Brien, Andrew Papachristos, and Zirui Song for helpful comments and suggestions. 


\section{Introduction}

In our paper, "Police killings and their spillover effects on the mental health of black Americans: a population-based, quasi-experimental study,"1 we sought to understand the role of structural racism as a driver of population health disparities. We operationalized this aim by correlating survey data on poor mental health days among people in the general U.S. population (obtained through the U.S. Behavioral Risk Factor Surveillance Survey) with officer-involved killings of unarmed black Americans (compiled by the Mapping Police Violence [MPV] project) occurring in the same state as the interview in the three months prior to the survey date. Our key finding was that officer-involved killings of unarmed black Americans were associated with worsened mental health among black Americans in the general U.S. population in the three months after those events, and that the observed effects were specific to those exposures and population subgroups. Our paper was published in The Lancet and released online on June 21, 2018. Replication data and code were also made publicly available on June 21, 2018.

On October 10, 2018, we were notified by the Lancet Correspondence Editor that a letter had been submitted by M. James Lozada and Justin Nix raising questions about the validity of the data on officer-involved killings: namely, that MPV's "very liberal definition of unarmed"2(p.1412) could yield inflated estimates of unarmed killings as well as inflated estimates of their impacts on population mental health. We submitted our response to the Lancet on October 19, 2018, arguing that eyewitness accounts are likely to be at least as important as police reports in shaping perceptions of officer-involved killings at the community level. On November 26, 2018, we were notified that our reply had been accepted for publication. The letter by Lozada and Nix was published, ${ }^{2}$ along with our reply, ${ }^{3}$ on April 6, 2019.

On December 30, 2019, Nix and Lozada (hereafter NL) submitted to SocArXiv a critique of our paper and re-analysis of our replication materials. ${ }^{4}$ NL disagreed with the coding of armed/unarmed status in the MPV database, and proposed recoding several classes of officerinvolved killings originally coded by MPV as unarmed. They reanalyzed the "corrected" (p.5) data after reclassifying these cases following their preferred criteria, reporting that they "failed to replicate any of the results presented in Bor et al." (p.5).

\section{Critiques and Responses}

We disagree with NL's argument about the suitability of the MPV data for our analysis. The MPV project has comprehensively documented officer-involved killings in the U.S. since 2013 and is the only crowd-sourced database in the field that has tracked the armed/unarmed status of decedents since 2013. The MPV data matched the time frame for our analysis, which coincided with the genesis of the Black Lives Matter movement. Finally, we believed that the separation of exposure assessment from analysis would limit the potential for researcher bias in coding, and we made sure to respect this distance throughout our work.

Standing in stark contrast to our perspective, NL described the MPV data as "compiled by activists who may not view these incidents through an objective lens" (p.5). NL identified 93 cases of officer-involved killings of unarmed black Americans in which they believe that decedents were incorrectly coded as being unarmed. This characterization wrongly implies 
inaccuracies in the coding of these 93 cases. Rather, the larger issue is that MPV employs a broad definition of unarmed status (Table 1). ${ }^{\dagger}$ In his comment on NL, Sinyangwe ${ }^{5}$ argued that these 93 cases were not "misclassified" but rather appropriately reflected MPV's approach to the coding, with some MPV decisions arguably more conservative than alternative data curation efforts and other MPV decisions arguably less conservative. In recoding the data, NL explicitly privilege the claims of officers over those of bystanders and other eyewitnesses (e.g. "toy/replica firearms or objects that might reasonably resemble deadly weapons to officers in the moment" [p.4]). In our opinion - and contrary to the perspective adopted by NL - the community-based origin of the MPV data was a strong impetus for using these data to understand "the impact of events widely perceived to reflect structural racism on the mental health of black Americans"1(p.308).

NL conducted two analytic exercises. First, they removed 52 cases of decedents they believed were not killed intentionally by on-duty police officers and recoded as "armed" 14 cases of decedents who were killed intentionally by on-duty police officers. Second, they additionally recoded as "armed" 25 cases of decedents who were documented as being in possession of a toy/replica firearm and 2 cases of decedents who were documented as being in possession of an object they believed "might reasonably resemble deadly weapons to officers in the moment" (p.4). NL mistakenly described their analytic exercise as a "replication." It is, rather, more appropriately described as a "reanalysis."

After recoding these 93 cases of officer-involved killings according to their preferred definition, NL found that their reanalysis yielded different point estimates. In their reanalysis \#2 - which excludes all 93 cases - they recovered an estimated regression coefficient of $b=0.09(95 \%$ confidence interval [CI], -0.03 to 0.20$)$. Notably, their preferred estimate lies within, and their preferred confidence interval significantly overlaps with, our estimated confidence interval $(b=0.14 ; 95 \%$ CI, 0.07-0.22).

We recognize that other investigators may have preferred a coding scheme different from either MPV or NL. Recoding as armed or unarmed the decedents in the seven categories of cases described by NL as "misclassified" yields $2^{7}=128$ different combinations of exclusions. The 128 possibilities range from including all seven categories (i.e., following the MPV coding, as we did in our paper) to excluding all seven categories (i.e., the coding preferred by NL in their reanalysis \#2). We fitted ordinary least squares regression models (corresponding to our primary analysis) to these 128 different datasets.

Figure 1 represents the distribution of estimated regression coefficients and confidence intervals from these 128 regressions. ${ }^{\star}$ As can be observed in the Figure, all of the point estimates are

\footnotetext{
† In our Lancet paper, we omitted as exposures officer-involved killings where the armed status of the decedent was coded as "unclear" or "vehicle." We also omitted exposures for which the race/ethnicity of the decedent was listed as "unknown."

¥ The decedents in two cases disputed by NL - Freddie Blue and Dominic Hutchinson - were coded by MPV as "unclear" in the October 21, 2016 database extract we used. Thus, the decedents in these cases were never considered as "unarmed" in any of our analyses. Using the remaining 91 cases disputed by NL, we were able to replicate their reanalysis point estimates and $95 \%$ confidence intervals. However, the sample size we obtained using their preferred coding scheme was 98,186, which differs from our original analytic sample of 103,710. NL did not report their sample sizes. Nor are we aware of any code and data posted to replicate their reanalysis.
} 
positive, consistent with the hypothesis that officer-involved killings of unarmed black Americans are correlated with poorer mental health among black Americans in the general U.S. population in the three months after those events. The 128 estimated regression coefficients range in value from 0.04 to 0.20 , with 110 (85.9\%) estimates lying within the estimated confidence interval reported in our primary analysis. Of these, 38 (29.7\%) estimates exceed the magnitude of that which we reported. In comparison, the estimated regression coefficient reported by NL in their reanalysis \#1 is the third smallest in the distribution, and their reanalysis \#2 estimate lies at the 30th percentile of the distribution. All of the estimated confidence intervals overlap significantly with the estimated confidence interval reported in our primary analysis. In addition, 78 (60.9\%) estimates would be considered statistically significant at the $\mathrm{p}<0.05$ level.

\section{Conclusions}

Empirical researchers must make a number of choices about how officer-involved killings should be classified and should be explicit about these choices. MPV are explicit about their coding decisions and have made all of their source data publicly available. Our replication archive, also publicly available, allows other investigators to interrogate contrasting perspectives and explore the potential implications for population health disparities. The findings reported in our original paper were robust to a number of specification checks and sensitivity analyses. It is unsurprising that point estimates differ when recoding the exposure variable. Given the theoretical framework for our paper, we stand by our decision to use the MPV database, rather than an alternate coding scheme such as that proposed by NL, which privileges officer claims over other communitygenerated information. Nonetheless, the findings reported here in our reply to NL demonstrate that our original substantive findings are additionally robust to a number of alternative coding schemes for the exposure.

\section{References}

1. Bor J, Venkataramani A, Williams D, Tsai A. Police killings and their spillover effects on the mental health of black Americans: a population-based, quasi-experimental study. The Lancet 2018; 392: 302-10.

2. Lozada M, Nix J. Validity of details in databases logging police killings. The Lancet 2019; 393: 1412-3.

3. Bor J, Venkataramani A, Williams D, Tsai A. Validity of details in databases logging police killings - authors' reply. The Lancet 2019; 393: 1413.

4. Nix J, Lozada M. Do police killings of unarmed persons really have spillover effects? Reanalyzing Bor et al. *2018”. SocArXiv 2020; (Jan 1): doi:10.31235/osf.io/ajz2q.

5. $\quad$ Sinyangwe S. Justin Nix and James Lozada are Wrong About Mapping Police Violence. Here's Why. Medium.com; January 4, 2020. https://medium.com/@samswey/justin-nix-andjason-lozada-are-wrong-about-mapping-police-violence-heres-why-95ab2194831d

6. Clemens M. The meaning of failed replications: a review and proposal. Journal of Economic Surveys 2017; 31(1): 326-42. 
Table 1. Categories of "Unarmed" Decedents in the Mapping Police Violence Database

Not holding any objects or weapons when killed

Holding household/personal items that were not used to attack others (cellphone, video game controller, cane, etc)

Holding a toy weapon (BB gun, pellet gun, air rifle, toy sword)

An innocent bystander or hostage killed

A pedestrian or motorist accidentally hit by a police car or passengers in a vehicle chased by police with no weapon on them

Drivers or passengers accidentally hit by a police car

A person who dies in police custody after a police use of force or police neglect of their medical needs

Alleged to be armed by the police, but multiple independent witnesses maintain the person was unarmed, video evidence shows that the person was unarmed, and/or circumstances indicate it was physically impossible for that person to be armed (i.e. claiming a person shot themselves with their own gun while handcuffed and under surveillance in police custody after being searched for weapons)

Notes: Definitions obtained from https://mappingpoliceviolence.org/aboutthedata on January 13, 2020. 
Figure 1. Distribution of Regression Coefficients and Confidence Intervals from 128 Regression Models Using Different Combinations of Exclusions of the Seven Categories Defined by NL

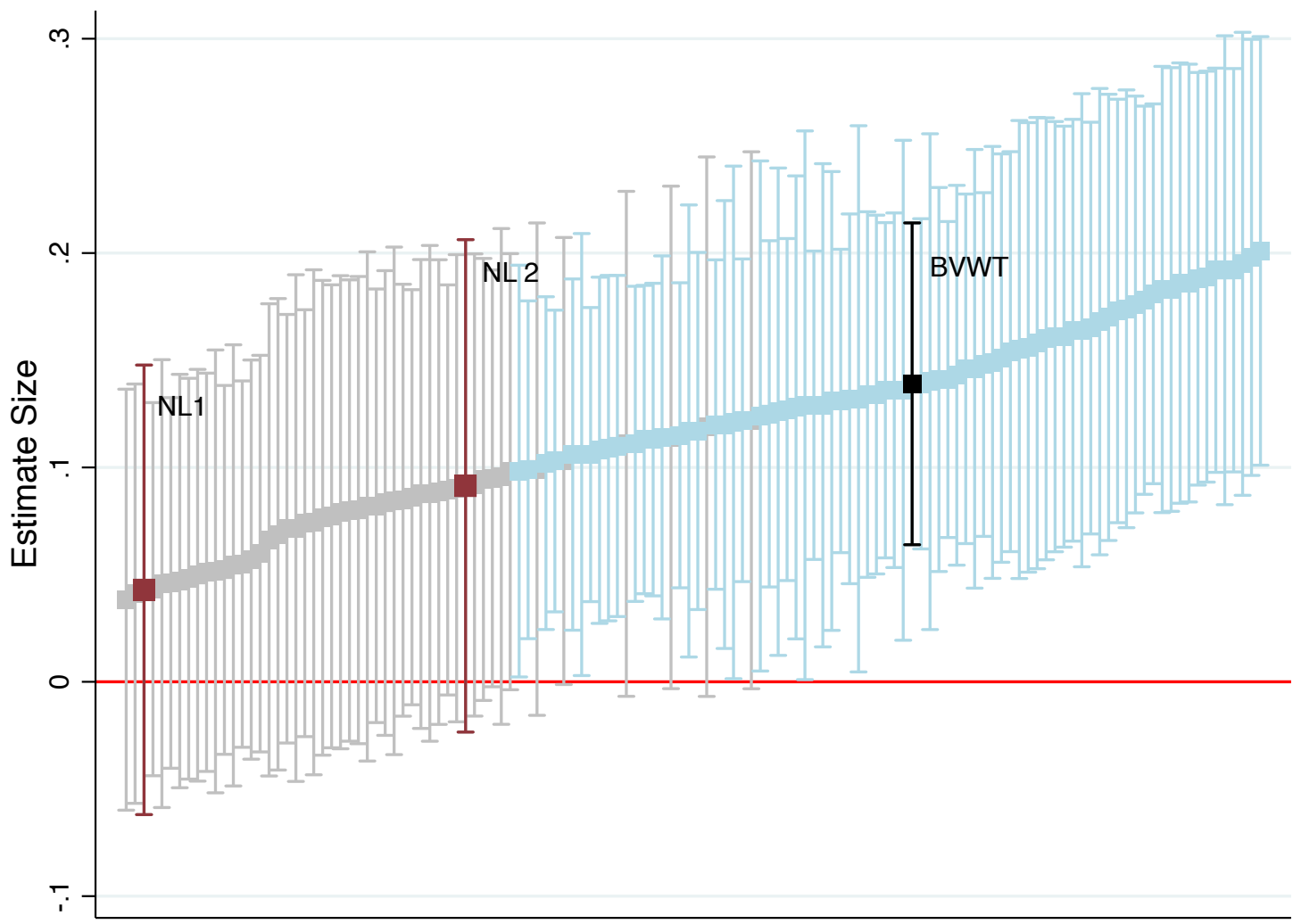

Notes: This caterpillar plot illustrates all regression coefficients obtained from fitting the ordinary least squares model in our primary analysis to 128 versions of the data, reflecting all of the different exposure terms constructed from all combinations of omissions of the 7 categories of cases disputed by NL. All models specify our primary outcome (number of poor mental health days among black Americans) and our primary exposure (number of officer-involved killings of unarmed black Americans in the same state in the 3 months preceding outcome assessment), adjusting for individual demographic characteristics, and state-month and month-year fixed effects. The coefficient and confidence interval labelled "BVWT" indicates our original finding. The coefficients and confidence intervals labelled "NL1" and "NL2" refer to the NL estimates obtained from excluding a subset of (\#1), or all (\#2), disputed cases. The confidence intervals in red overlap with zero. 